\title{
Gravitational Wave Detectors on Earth
}

\author{
Piero Rapagnani \\ Università La Sapienza and INFN, Sezione di Roma, Piazza Aldo Moro 5, Roma, Italy \\ E-mail: piero.rapagnani@roma1.infn.it
}

\begin{abstract}
Since the pioneering work of Joseph Weber, the quest for the detection of gravitational waves has progressed at higher and higher levels of ingenuity and effort. Here we shall briefly outline the historical path followed during the development of the detectors on ground, which have brought us from the resonant bars to the large interferometric antennas of today, and give some perspective on the exciting time that is probable to come, when the large interferometric detectors will have completed their upgrade to the Advanced stage, with a sensitivity ten times better than the present one. At that time, about 2015, several events per year should be detected. On the long term scale, more sensitive gravitational detectors, currently in the conceptual design phase, will start a systematic gravitational wave astronomy.
\end{abstract}

\section{Introduction}

As we know from cosmology, most of the matter in the universe interacts only though the gravitational field. On the other hand, gravitational interaction is still the less known fundamental force of nature, and its integration in a unified picture still seems to be far away. A crucial step toward the inclusion of gravity within a unified theory of all interactions could be the detection of gravitational radiation. Such an event will provide a unique way to observe the behavior of matter and gravitational field at energies and densities unreachable by any laboratory experiment.

The first attempt at detecting gravitational waves dates back at the beginning of 1960's, when Joseph Weber started the field. Weber's detectors had their peak sensitivity at frequencies around $1 \mathrm{kHz}$, with a bandwidth of only a fraction of Hz. Today, as we shall see in the following, large interferometric antennas have sensitivities many orders of magnitude better, and a bandwidth of several $\mathrm{kHz}$ starting from $10 \mathrm{~Hz}$. Moreover, the importance of the scientific goal was such that many different experiments and detectors have been conceived over the years and are being proposed today to cover almost the entire gravitational wave spectrum, much as it has happened for e.m. radiation.

Indeed, though the aim of this article is to outline the progress of detectors on ground, we think it is essential to give a hint of the huge effort being carried to try to detect gravitational waves.

Thus, in Table 1 we report some expected sources of gravitational waves from the lowest to the highest frequency range, with the related detectors and an indicative reference. At frequencies as low as $10^{-16} \mathrm{~Hz}$ cosmological models predict a relic gravitational wave radiation [1] that could be detected by Cosmic Microwave Background (CMB) experiments measuring the polarization the background e.m radiation. In the range of frequencies between $10^{-9}-10^{-7} \mathrm{~Hz}$ pulsar timing is being used since 1980's [2] to detect gravitational waves coming, for instance, from binary 
Super Massive Black Holes (SMBH) in galactic nuclei. At higher frequencies, $10^{-4}-10^{-1} \mathrm{~Hz}$ the LISA [3] space mission is being developed. LISA will be a constellation of six drag-free satellites, with two at each vertex of an equilateral triangle having a side of $510^{6} \mathrm{~km}$ that will fly in formation at $1 \mathrm{AU}$ from the Sun and 20 behind the Earth. Three subsets of four adjacent spacecraft each form a Michelson interferometer comprising a central station, consisting of two relatively adjacent spacecraft (200 km apart), and two spacecraft placed at a distance of $5 \times 10^{6}$ $\mathrm{km}$ from the centre to form arms which make an angle of $60^{\circ}$ with each other. The technologies relevant for LISA will be tested in the LISA Pathfinder satellite [4], that will be launched in the near future. Once operative, sometime after 20202, the LISA detector will be so sensitive to be signal dominated: the real challenge of LISA data analyisis will be to distinguish a single object from the continuous gravitational wave background. Current interferometers have sensitivities starting form $10 \mathrm{~Hz}$. The gap between LISA and laser antennas on ground could be filled in the future by the DECIGO [6], a Fabry-Perot interferometer in space with arms of $1000 \mathrm{~km}$ length, with sensitivity in the range $10^{-1}-10 \mathrm{~Hz}$, or by the starting new technology of atomic interferometers [7].

After forty years since it was born, the field of gravitational wave detection is still growing, building instruments of increasing complexity and ingenuity that should be able to detect the first real signals in the near future: the next years will be a very exciting time for groups looking for gravitational radiation.

Table 1. Spectrum of Gravitational Wave Sources

\begin{tabular}{|c|c|c|c|}
\hline Freq. Range $(\mathrm{Hz})$ & Source & Detector & Reference \\
\hline$\simeq 10^{-16}$ & $\begin{array}{l}\text { Quantum fluctua- } \\
\text { tions of very early } \\
\text { universe }\end{array}$ & $\begin{array}{l}\text { Polarization map } \\
\text { of CMB }\end{array}$ & [1] \\
\hline $10^{-9}-10^{-7}$ & $\begin{array}{l}\text { Binary Supermas- } \\
\text { sive BH in galac- } \\
\text { tic nuclei (SMBH) }\end{array}$ & $\begin{array}{l}\text { Precision timing } \\
\text { of millisecond } \\
\text { pulsars }\end{array}$ & {$[2]$} \\
\hline $10^{-4}-10^{-2}$ & $\begin{array}{lr}\text { Black } & \text { Holes, } \\
\text { Compact } & \text { Stars } \\
\text { captured } & \text { by } \\
\text { SMBH } & \end{array}$ & $\begin{array}{l}\text { Laser tracking } \\
\text { spacecraft array } \\
\text { (LISA) }\end{array}$ & [3] [4] \\
\hline $10^{-2}-10$ & $\begin{array}{l}\text { Binary stars in } \\
\text { the galaxy and } \\
\text { beyond }\end{array}$ & $\begin{array}{l}\text { Big Bang Ob- } \\
\text { server, DECIGO } \\
\text { space detectors, } \\
\text { atomic interfer- } \\
\text { ometers }\end{array}$ & {$[5][6][7]$} \\
\hline $10-\simeq 10^{4}$ & $\begin{array}{l}\text { Merging Binary } \\
\text { NS, BH, fast } \\
\text { pulsars }\end{array}$ & $\begin{array}{l}\text { Resonant Bars, } \\
\text { Large Interfero- } \\
\text { metric Detectors }\end{array}$ & See ref. in this paper \\
\hline
\end{tabular}




\section{A brief overview of gravitational wave sources}

Before to start the description of the evolution of ground gravitational detector, it is useful to give a brief description of the most relevant sources of gravitational waves that these experiments are trying to detect. Indeed, much of the historical evolution of these instruments is due to the evolution of the theoretical predictions concerning the most powerful and frequent sources.

As it is said in the introduction, the typical sensitivity bandwidth starts at around $10 \mathrm{~Hz}$ and ends at 5-6 kHz. This range determines also the kind of sources that can be detected. They are usually divided in four categories:

(I) Emission due to Compact Binary Coalescences $(C B C)$ : this sources are coalescing binary systems [8], where each component of the system can be a Neutron Star (NS) or Black Hole $(\mathrm{BH})$. It is possible to model the inspiral of the two compact objects with great precision, and so matched filtering can be used in the analysis, increasing the detection efficiency.

(II) Burst sources: sources like core-collapse Supernovae [9], merging Black Holes, or quakes in the crust of Neutron Stars cannot be modeled with sufficient precision to use matched filtering. In this case more general analysis techniques must be used.

(III) Continuous wave sources: continuous gravitational waves are emitted by rotating Neutron Stars with non zero eccentricity $\epsilon$ [10]. The maximum signal that could be emitted by a Pulsar depends on the maximum eccentricity allowed by the equation of state of the star. In standard models, the energy lost by a Pulsar for gravitational wave emission is only a small fraction (less then 1\%) f of the overall energy lost by the star, which is measured by its spindown rate.

(IV) Gravitational Wave Background: cosmological models predict the existence of a wide band stochastic background of continuous gravitational waves [11]. The detection of a stochastic background would be quite important to distinguish between different cosmological models.

\section{The importance of the Network}

Since the start of this field of research, the importance of having multiple detectors operating in coincidence has been evident. Weber, in his pioneering work [12], developed for the first time two identical resonant antennas at room temperature, located at a distance of about $1000 \mathrm{~km}$, so to be able to analyze the data and find if signals were present at the same time in both antennas. This concept has remained and has grown in depth and scope as the field progressed: the network is the real detector, and a detector whose sensitivity is limited by the less sensitive of its components. So, on one side, the competition to build the best detector has been always very strong in the field, while on the other hand a very strict collaboration between different experimental groups has always been necessary, though sometimes made rather difficult by the understandable wish by experimentalists to protect and promote their own apparatuses so carefully and painfully built.

In the following sections, we shall briefly outline how the Network of ground detectors evolved with time, and which are the main limits to the sensitivity of the apparatuses that have been and will be part of it.

\section{The resonant detectors}

In the 1960's, following Weber's claim to have detected bursts of gravitational waves, several groups tried to duplicate and improve his experiment with resonant detectors at room temperature [13]. Networks of at least two detectors with sensitivities similar to Weber's antennas were built and operated all over the world, and computer analysis of the data was introduced, and became with time more and more complex.

As it is well known, the early results of Weber were not confirmed. It was soon realized that a further step was necessary: the sensitivity of the antennas had to be greatly improved, at least by a factor $10^{3}$, so to make signals at the level of those observed by Weber quite evident, 
and, eventually, be able to observe similar signals at a longer range. This sensitivity goal could be reached using larger detection mass with respect to Weber's antennas $(2500 \mathrm{~kg}$ in place of $1500 \mathrm{~kg}$ ), lowering the thermal noise by cooling the big aluminum bars down to liquid helium temperature, $4.2 \mathrm{~K}$, and using the lowest noise amplifier available, a dcSQUID, to detect the vibrations of the antennas due to the signal.

The Network had to consist of at least three antennas, widely separated one from the other, so that their signal would bear no correlation due to local disturbances, and also, in case of detection, some time correlation analysis could be used to find direction and velocity of the incoming waves.

So, in 1971, an agreement was signed by William Fairbanks of the University of Stanford, by William Hamilton, of the University of Louisiana, and Edoardo Amaldi and Guido Pizzella of the University of Rome "La Sapienza", to build three large cryogenic resonant antennas, to be constructed in about five years and be operated in coincidence. As it came out, this prediction was optimistic: more than fifteen years of hard work were necessary to reach the sensitivities set as a goal by that agreement. In 1986 the three cryogenic antennas in Stanford, Baton Rouge (the ALLEGRO antenna built by the Louisiana State University group), and at CERN in Geneva (the Explorer antenna built by the Rome group) run in coincidence for three months from april to july [14]. The sensitivity of those detectors was of the order of an adimensional strain amplitude $\frac{\Delta l}{l}=h \simeq 10^{-18}$ over a bandwidth of $\simeq 0.5 \mathrm{~Hz}$ centered around $1 \mathrm{kHz}$.

In the following years, the cryogenic resonant detectors run in coincidence for longer and longer periods. Unfortunately, the Stanford group withdrew from the field, after the untimely death of William Fairbanks and as a consequence of the damage of their detector during the earthquake of october 17th 1989. However, two other groups joined the search: the group led by Joe Blair at the University of Perth in Australia, which built the Niobi cryogenic antenna, and the group led by Massimo Cerdonio of the University of Padova that built the AURIGA detector. AURIGA was part of a new generation of resonant detectors, that used He3-He4 dilution refrigerator to cool down the $2500 \mathrm{~kg} \mathrm{Al}$ bar to $0.1 \mathrm{~K}$, with a further reduction of thermal noise. This new technique has been pioneered by the NAUTILUS detector, which was designed and assembled at CERN by the Rome group, before being moved to its final destination, the National Laboratory of INFN in Frascati, Italy. In february 1991, NAUTILUS was cooled down for the first time and became the first cryogenic antenna to reach a temperature as low as $97 \mathrm{mK}[2]$, a technological achievement in itself.

In the 1990's, after many years of continuos progress and refinements, the resonant antennas reached typical sensitivities better than $h \simeq 10^{-19}$ in a bandwidth of some $\mathrm{Hz}$ centered around $1 \mathrm{kHz}$, with a duty cycle of about $95 \%$ and a rate of spurious signals almost negligible (one false alarm per century).

In that period, the groups of resonant bars in operation organized themselves in the IGEC: International Gravitational Event Collaboration, to manage the exchange and analysis of the data of the respective detectors. After more than twenty years, the network of cryogenic and ultra-cryogenic detectors imagined by the Rome-LSU-Stanford agreement had reached its goal. The results of this analysis are in [16] for the period 1997-2000 and in [17] for the period from 2005 to 2006. No triple coincidences were found. The final upper limits set on the gravitational wave events rate is shown in Figure 1. They refer to the kind of signal that resonant bars can observe with their best sensitivity: gravitational wave bursts centered at about $1 \mathrm{kHz}$ and of only some milliseconds duration. When the resonant bars were designed and constructed, the most promising sources of gravitational waves predicted by theory were indeed class II SNe. It was hoped at that time that a highly asymmetrical collapse could convert in gravitational waves the equivalent in energy of $10^{-5}-10^{-6}$ solar masses. Unfortunately nowadays collapse models, refined over the years, predict that only a fraction as low as $10^{-8}$ solar masses is emitted as gravitational waves. This means that current resonant detectors can only observe SNe events 


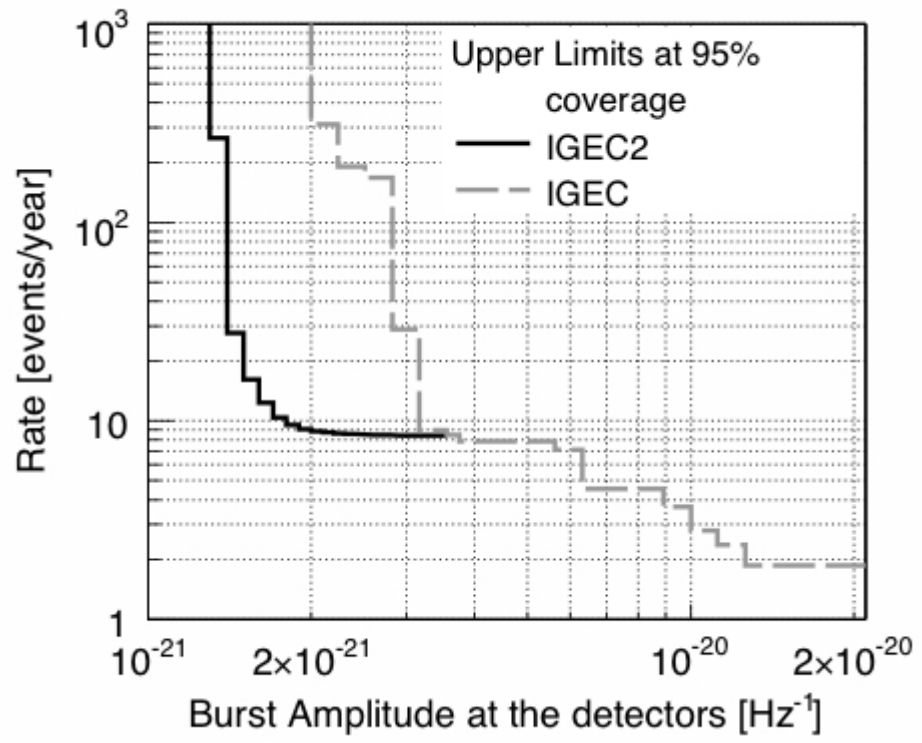

Figure 1. Upper limits on the rate of gravitational wave burst at $1 \mathrm{kHz}$ set by the IGEC and IGEC-2 analysis.

occurring in our galaxy. The rate of these events is expected to be $0.04 \mathrm{SNe} /$ year, i.e. about one every 25 years. Though very rare, the observation of such an event, eventually in coincidence with e.m. and neutrino (see for instance [18]) observatories, would be quite relevant. Thus, both Auriga and Nautilus antennas are still in operation, permanently on watch in case that a $\mathrm{SNe}$ event in our galaxy is detected, covering with their observations the periods when all other gravitational wave antennas in the world are offline,

\section{The beginning of gravitational interferometers}

The contribution of Resonant Bars has been essential in establishing the field and putting some important first upper limits on the gravitational landscape around us, but now the hope for detection is in the Network of long arm interferometers. Indeed, as theoretical models of gravitational wave emission progressed, it became more and more evident that the most powerful and frequent events would be the coalescence of binary systems of two compact objects, Neutron Stars or Black Holes. These signals have a strong signature, because their frequency increases rapidly as the two compact object approach one another. Templates of the waveforms of the gravitational waves emitted during this inspiral stage can be computed with good precision up to the last phase, when the two compact objects merge together (the merger phase) and the computation becomes quite difficult. It is clear that CBC signals can be detected more efficiently if the inspiral can be followed for a longer time, that is, if the detector has a large bandwidth. So, with time, bandwidth became an essential parameter in the development of gravitational wave antennas.

The idea to use an interferometer to detect gravitational radiation was first pioneered in the 1970's by Edward L. Forward (see for instance [19], [20]). This new detection concept promised far greater sensitivities than the bar detectors and quite large bandwidths: indeed an interferometer is an intrinsically wide band detector, while in a resonant bar it is very difficult to develop technologies allowing comparable bandwidths [21] (recently, new ideas for a large 
bandwidth bar detector has been proposed [22], and the basic principles tested, but the first real proposal is still in progress). However, the potential sensitivity of the new interferometric detectors had to be confronted with technological difficulties: at that time the level of knowledge on lasers and optics was not sufficient to build instruments that could compete with resonant bars. Nevertheless, several groups around the world started to develop interferometers as gravitational antennas. The main groups were in Europe (Germany and United Kingdom), USA, and Japan. In the 1990's, the Japanese groups started to build TAMA, a $300 \mathrm{~m}$ arms interferometer at Mitaka, Tokyo [23]. This machine included all the technologies necessary to build far larger interferometers: e.g. mechanical filters to suspend optics avoiding seismic noise, Fabry-Perot resonant cavities, and a resonant optical cavity to enhance the laser power injected into the interferometer (the power recycling) [?]. The successful start of operation of this interferometer, in 1998, proved for the first time that the technological choices at the core of these detectors were correct [25]. Today, TAMA is still in operation, testing technologies for future interferometers.

In Europe, in 1975 the Max Planck Institute for Astrophysics in Munich started with a prototype of $3 \mathrm{~m}$ length, which in 1983, at the Max Planck Institute of Quantum Optics (MPQ) in Garching, led to a prototype with 30 m length [26]. In 1977 the Department of Physics and Astronomy of the University of Glasgow began also to study laser interferometry for gravitational radiation detection [27], and in 1980 started operation of a $10 \mathrm{~m}$ prototype. In 1985 the Garching group proposed the construction of a large detector with $3 \mathrm{~km}$ arm, the British group an equivalent project in 1986. The two groups combined their efforts in 1989 proposing GEO, a large interferometer to be built in the Harz mountains (Northern Germany). However, the project could not be funded, because of financial problems. Thus in 1994 a smaller detector was proposed: GEO600, with arms of $600 \mathrm{~m}$ in length, to be built near Hannover. The construction of the new detector started in September 1995 and stable operation began in 2002 [28]. The teams working on GEO600 made the particular choice to include in their machine many advanced technologies that could be used to enhance the sensitivity of the larger interferometers currently in operation or being built in USA and Europe. For instance, in GEO600 two particular technologies have been tested, that were not present at the starting stage of km-length interferometers, and will be implemented, as illustrated in the following, only in the Advanced Detectors: signal recycling to reduce the shot noise [30] and monolithic suspensions to reduce thermal noise [29]. Moreover, GEO600 is now playing a central role in the development of squeezed laser light, a technique that reduces the quantum phase noise of the laser light inside the interferometer [31]. Also this technique will be used in the following years on future large detectors.

\section{The Large Gravitational Interferometers Network}

The experience gained with short arm interferometers was essential for the development of kmsize detectors, that could take the lead in the field and do real science. In USA, groups at Caltech and MIT joined forces to build LIGO [32] a large observatory consisting of three gravitational wave interferometers, with arms of $4 \mathrm{~km}$, in two sites about $3000 \mathrm{~km}$ one from the other: the LIGO Observatory in Hanford, Washington State and the LIGO Observatory in Livingston, Louisiana. The LIGO detectors started to operate in 2002 and reached their design sensitivity in 2007, after five Science Runs (see Fig.4). Though the size of the experimental effort behind these machines is orders of magnitude greater than the initial experiments set up by Weber, as quite different are the experimental techniques, the network principle is the same as it was at the beginning of the field. Indeed, LIGO was the first network of large interferometers to go on air and probe the space around us at unprecedented distances and frequencies, setting a series of new upper limits on all kinds of gravitational sources [33], [34].

In particular, in [34], mass-dependent upper limits on the rate of $\mathrm{CBC}$ events are reported, 
expressed in terms of $L_{10}$ units, where $L_{10}$ is $10^{10}$ times the blue solar luminosity (the Milky Way contains $\simeq 1.7 \mathrm{~L}_{10}$ ). For instance, for NS-NS inspirals, a rate upper limit is set of $3.8 \times 10^{-2} \mathrm{yr}^{-1} L_{10}{ }^{-1}$, still significantly higher than recent $\mathrm{CBC}$ rate estimates derived from the observed Binary NS population, $5 \times 10^{-5} \mathrm{yr}^{-1} L_{10}{ }^{-1}$ [35].

As far as gravitational wave bursts are concerned, according to [34] the all sky search of the complete S5 Science Run will allow to put upper limits on signals as low as $5 \times 10^{-8}$ solar masses occurring at a typical Galactic distance of $10 \mathrm{kpc}$. If we move to the Virgo galaxy cluster, approximately $16 \mathrm{Mpc}$ away, the minimum detectable energy increases to 0.12 solar masses.

More interesting is the triggered search for bursts events, where the analysis of detector data is related to observations of supernovae, anomalous optical transients, radio bursts and neutrinos. The constraints on position, time and signature given by these external triggers allow to increase the sensitivity of the search and to gain useful information also in the case of a null result.

For instance, in [36] it is reported the analysis of gravitational wave data from LIGO, coincident in time with the exceptionally intense GRB 070201. The position in the sky of this gamma ray burst overlapped one the spiral arms of the nearby galaxy Andromeda (M31). The data showed no evidence of an inspiral or a more general burst signal; this result ruled out (at the $99 \%$ level) the possibility of a binary merger in M31 as the origin of GRB 070201.

In Europe, in the same period, several groups of experimentalist in France and Italy started to build Virgo, an interferometer with arms of $3 \mathrm{~km}$, located in Italy in Cascina, near Pisa. Today, Virgo is the only interferometer having a suspension technology [37], that allows a good sensitivity starting from frequencies as low as $10 \mathrm{~Hz}$. With this bandwidth, in the near future, the detection of continuous waves coming from rotating neutron stars could be accessible. These signals are unique in the sense that they have a very strong signature because of the Doppler shift as the position and relative velocity of the source with respect to the Earth change during a year. So, Virgo (and LIGO, starting from about $50 \mathrm{~Hz}$ ) could detect continuous gravitational waves coming from pulsars without any coincidence with other detectors. Indeed LIGO has already set some upper limits on gravitational radiation coming from pulsars. For instance, in [38] data from the fifth run LIGO detectors are used to set upper limits on the gravitational wave emission from 116 radio pulsars. In particular, in that paper an upper limit is reported for the Crab Pulsar 7 times lower than the fiducial spin-down limit, limiting the power radiated via gravitational waves to be less than $2 \%$ of the available spin-down power.

However, both LIGO and Virgo scientists are well aware that the Network is the real detector, and that a Network is as efficient as the collaboration between the groups that support it. As a consequence, in 2006 both Collaborations signed an agreement to perform scientific runs in coincidence and for a complete exchange of data and competences. Virgo started operation in 2003, and had its first scientific run VSR1 in 2007 [39], in coincidence for 4 months with LIGO S5 scientific run which had started in november 2005. Previously, in january 2006, also GEO600 had joined for a period the LIGO S5 run, giving its contribution to the extension of the network. Since than, Virgo and LIGO and GEO600 detectors had shared all data, and merged the groups doing the data analysis. The hope is that the collaboration of LIGO, GEO600 and Virgo will improve with time in front of the demanding tasks to reach the Advanced Detectors sensitivity goals.

One of the first scientific results of the joint analysis of LIGO and Virgo data is a new upper limit on the stochastic background of gravitational waves [?]. The new limit constrains the energy density of the stochastic gravitational-wave background, normalized by the critical energy density of the universe, in the frequency band around $100 \mathrm{~Hz}$, to be less than 6.9 ? 10?6 at $95 \%$ confidence, improving on the limits set by the Big Bang Nucleosynthesis and the Cosmic Microwave background. allowing to reject some models for the early evolution of the universe.

Moreover, data from the first Virgo Run and the fifth LIGO and GEO 600 run have been used to search for signals in temporal coincidence with 22 short GRB's [41] and 137 GRB's 


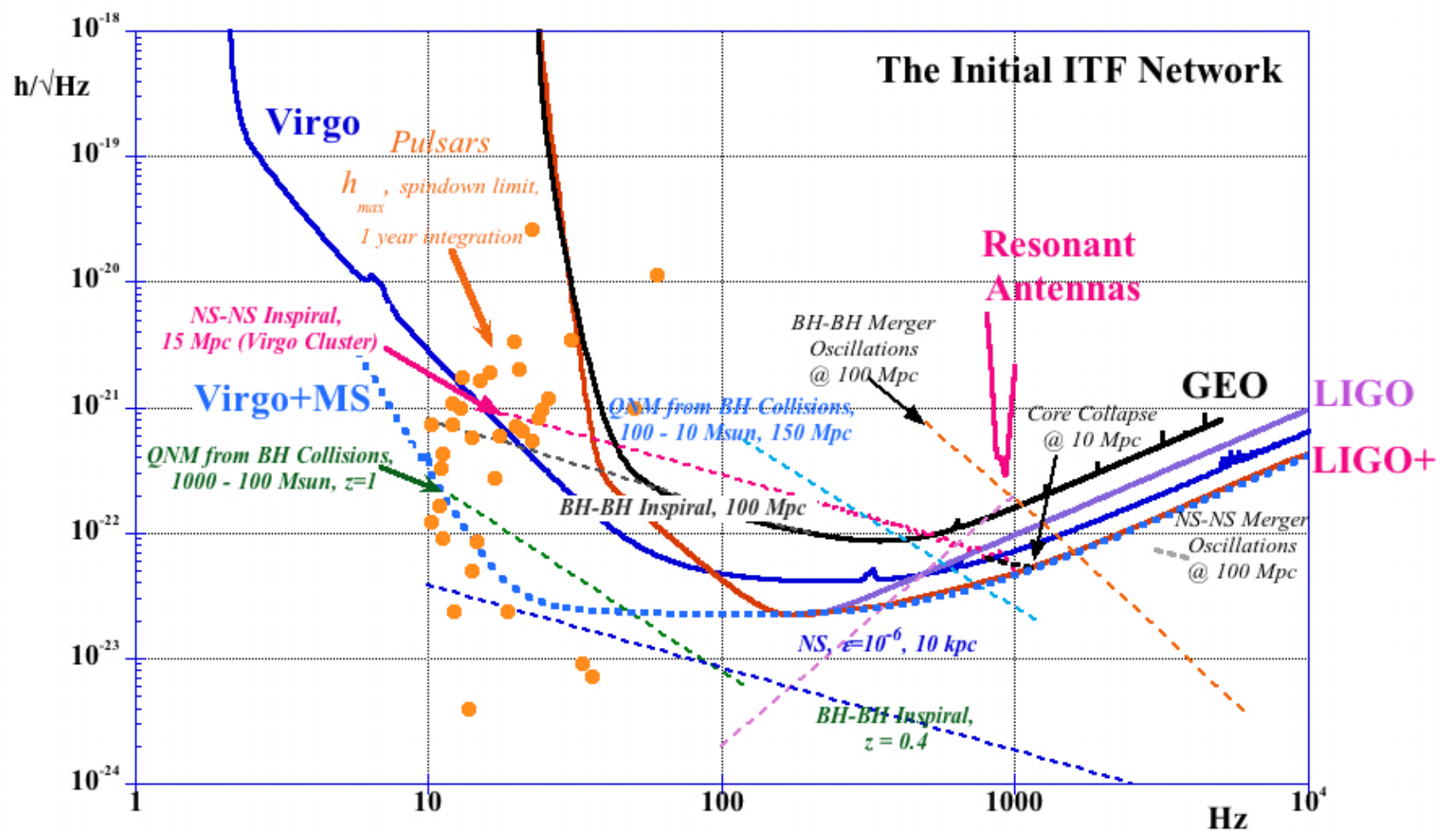

Figure 2. The sensitivity of the Initial Network of Interferometers, GEO600, LIGO and Virgo. The sensitivity of resonant bars is also plotted, together with some characteristic sources. Though some sources are above the sensitivity threshold of initial interferometers, their rate, as shown in Table 2, is still too small to be detected with observations of only a few years.

[42]. The search gave no positive result, but allowed to refine this triggered search in view of an increase in sensitivity of the detectors in the Network. Indeed, the coincidence search with GRB's, which are thought to be related to CBC and core collapses, is one of the most promising methods currently available to identify a gravitational wave event. In the current Network, the maximum distance at which these events are observable does not allow to cover a volume of universe large enough to be able to detect some events per year: a further increase in sensitivity is necessary, to be able to detect farther events. Each step of increased sensitivity means an increase of the available volume with the third power, and a related increase of the expected rate of gravitational events. These considerations are detailed in Table 2, where the rate of the most promising sources is reported as function of the range that can be reached by the Interferometer Network. As it can be seen, two further technological steps are being prepared: the Advanced Detectors stage (also referred to as second generation stage), and the Third Generation stage. As it can be seen from Table 2 [43], while the Initial Network sensitivity is not enough to expect appreciable rates, Advanced Detectors will be able to observe several events per year.

\section{The near future and the start of gravitational wave astronomy}

Both LIGO and Virgo are currently being upgraded to the Enhanced state, with a set of technologies that are a step toward the final sensitivity goal of these interferometers: the Advanced Stage.

The Advanced Detectors will have a sensitivity about ten times better than in the initial 
Table 2. Neutron Star and Black Hole Mergers Event Rate

\begin{tabular}{l|c|c|c|c|c|c}
\hline & \multicolumn{3}{|c|}{ Range (Mpc) } & \multicolumn{2}{c|}{ Rate } \\
\hline & NS-NS & NS-BH & BH-BH & NS-NS & NS-BH & BH-BH \\
Initial ITFs & 20 & 45 & 100 & $0.025-05$ & $0.001-01$ & $0.02-05$ \\
Advanced ITFs & 300 & 650 & 1500 & few to 1500 & $2-100$ & 50-1000's \\
3rd Generation ITFs & $\mathrm{z}=0.28$ & $\mathrm{z}=0.4$ & $\mathrm{z}=1$ & & & \\
\hline
\end{tabular}

stage. As written above, the technologies at the base of this upgrade are currently being tested in GEO600 (monolithic suspensions, signal recycling and squeezed light) and also in Virgo: the Virgo+MS upgrade [44], started in january 2010, has tested for the first time the suspension of large mirrors with monolithic fused silica fibers in a km-size interferometer, and is currently under commissioning. If successful, the Virgo+MS upgrade should allow to decrease the thermal noise almost at the level needed for Advanced Virgo. At the same time, LIGO is doing an intermediate upgrade, Enhanced LIGO [45], which will reach an increase in sensitivity testing at the same time some Advanced LIGO technologies, mainly related to the injection and detection systems. Enhanced LIGO and Virgo+MS should have a run in coincidence for some months in 2010.

By the end of 2010, LIGO will start its upgrade to Advanced [46], followed by Virgo in summer 2011 [47]. LIGO should start to take data again, at least with one interferometer, in 2013. Virgo should follow in 2015. At the advanced stage, the Network of detectors should be able to probe a volume of space about one thousand times larger than today: as shown in Table 2 , the rate of detection, at least for bursts coming from inspirals of binary massive objects, should be of the order of several events per year, and allow, with a high probability, the first detection of a gravitational wave.

In a similar way, the GEO collaboration is upgrading their detector to the GEO-HF phase [48], which will use signal recycling technology to reach sensitivities at high frequencies that would not be accessible to the advance detectors.

After these fundamental steps, the beginning of a systematic studies of astrophysical sources will require an improvement in detector sensitivities by another factor ten. Conceptual studies of such machines are beginning right now: recently the EU, in the frame of FP7, approved a three year program for a Design Study of a third generation gravitational wave detector [49], the Einstein Telescope (ET) project. ET will be located underground to reduce seismic noise, and will use cryogenic technology to cool down the suspended mirrors and reduce thermal noise. The limits given by shot noise will be overcome by a further increase in laser power and by using squeezed states of the injected light. The underground location will be essential also to overcome another fundamental noise limiting the sensitivity at frequencies around $1 \mathrm{~Hz}$ : the Newtonian noise, due to the motion of ground and air around the test masses.

As shown again in Table 2, the ET sensitivity will allow the study of the entire gravitational universe, detecting binary neutron star mergers within a red-shift of $\mathrm{z}=0.4$, binary black hole sources to within a distance of $\mathrm{z}=2$, and all milli-second period neutron stars in the Galaxy with ellepticities larger than $10^{-8}$.

A similar program, proposing to build a cryogenic interferometer underground, is being studied in Japan by the LCGT project [50]. The ambition of the LCGT Collaboration is to 


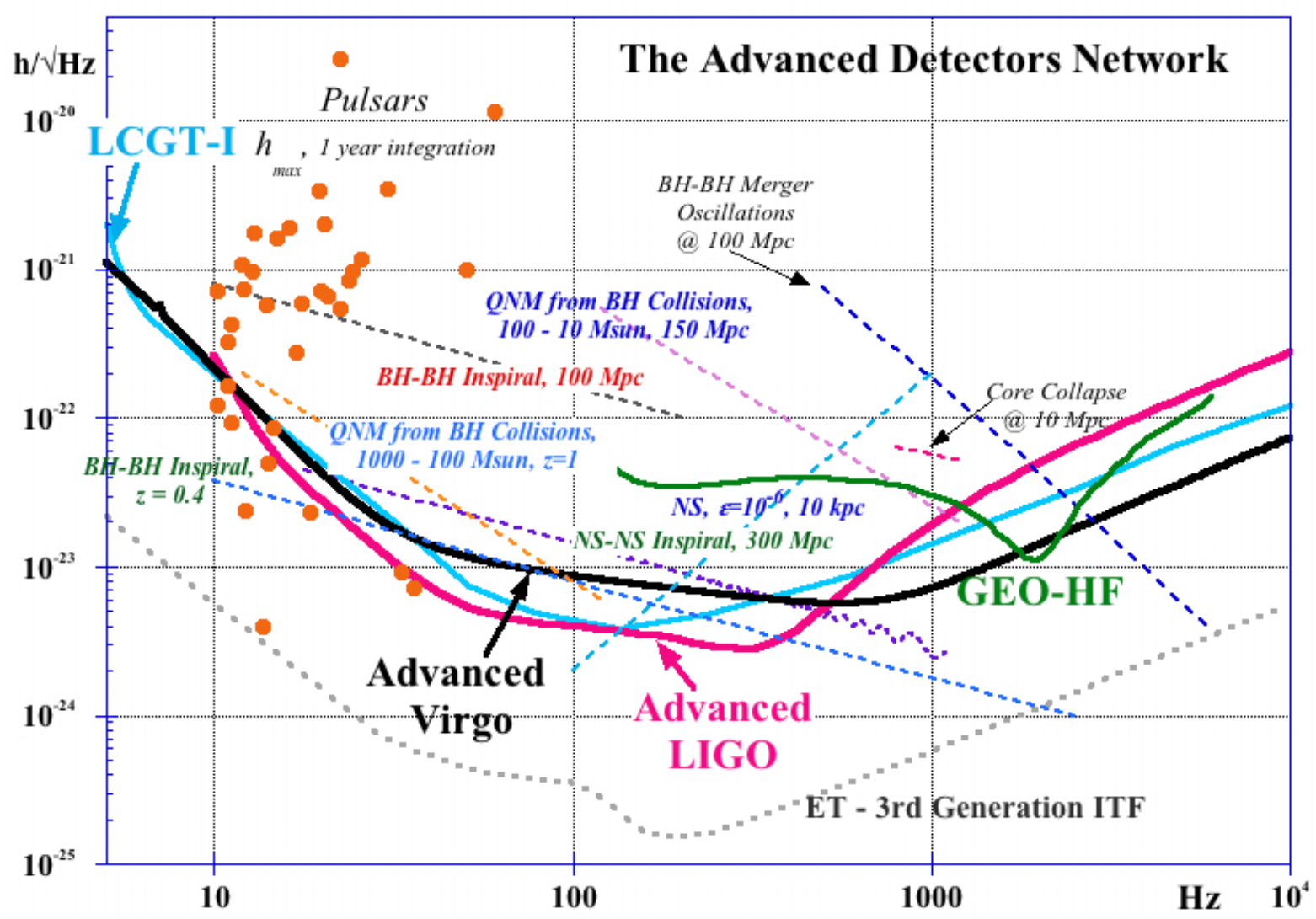

Figure 3. The predicted sensitivities of the Advanced Detector Network. At this stage the network should be able to detect several gravitational events per year. The expected sensitivity of a third generation detector is also shown.

put in operation a gravitational interferometer in a time frame similar to those of the Advanced Detectors, using third generation technology. The japanese groups have already taken a step in this direction with the construction of CLIO [?], a test interferometer with arms of $100 \mathrm{~m}$, having mirrors cooled down to about $20 \mathrm{~K}$ by pulse-tube refrigerators.

Recently (june 2010), the LCGT project has been approved by Japanese funding agencies: by 2014-2015 the collaboration will build an underground detector, to exploit the lower seismic noise underground, adding cryogenics in a second step. So, LCGT will join the Network of Advanced interferometers, increasing its efficiency in a very relevant way.

A network of third generation interferometers, dedicated to the systematic study of gravitational sources, could reach its maximum efficiency only including at least one observatory in the southern hemisphere. This could be one of the strongest advantages of the proposed AIGO detector [?], a gravitational interferometer to be built in Gingin, north of Perth in Western Australia.

If the advanced detectors will be successful in observing for the first time gravitational radiation, than the construction of a large Network of third generation gravitational observatories will be mandatory: together with the new instruments being currently developed for gamma and neutrino astronomy, the Third Generation Network will open up a completely new way to probe our universe. 


\section{Conclusions}

The history of the search of gravitational waves started in the 1960's with the pioneering work of Joseph Weber. After more than forty years of work, next years should finally see the first detection of a gravitational wave event by the advanced generation of Interferometric Detectors, Advanced LIGO and Advanced VIrgo. After that, the construction of large interferometers underground and using cryogenic techniques (the ET project in Europe, LCGT in Japan) should finally be the start of systematic gravitational astronomy.

\section{Acknowledgments}

It is more than forty years that the search for gravitational waves is being carried on: I think it is time to thank all those that worked to build gravitational wave detectors and are now retired or no more in the field. A number of physicists, engineers and technicians whose work is at the base of the machines being built today. I acknowledge also the useful suggestions and comments of the unknown referees.

\section{References}

[1] Crittenden, R. et al. Phys. Rev. Lett. 711993324

[2] Foster, R. S., Backer, D. C. The Astrophysical Journal 3611990 300-8

[3] Danzmann, K. and the LISA Study Team Adv. Space Res. 3272003 1233-42

[4] P McNamara, P. et al. Class. Quantum Grav. 252008114034

[5] Corbin, V. and Cornish, N.J. Class. Quantum Grav. 2320062435

[6] Sato, S. et al. Journal of Physics: Conference Series 1542009012040

[7] Tino, G. M. and Vetrano, F. Class. Quantum Grav.24 (2007) 2167-78

[8] Belczynski, K., Kalogera, V. and Bulik, T. Astrophys. J. 5722001 407-31

[9] Dimmelmeier, H., Font, J.A. and Muller, E. Astrophys. J. 5602001 L1636

[10] Jaranowski, P., Krolak, A. and Schutz, B.F. Phys. Rev. D 581998063001

[11] Maggiore, M. Phys. Rep. 3312000 283-367

[12] Weber, J. Phys. Rev. Lett 2219691320

[13] Levine, J. L. Physics in Perspective 6 (2004) 42-75

[14] Amaldi, E. et al. (1989) Astronomy \& Astrophysics (ISSN 0004-6361), 216, 325-332

[15] Astone, P. et al. European Physics Letters 16 (1991) 231

[16] Astone, P. et al. Physical Review D 68 (2) (2003) 022001

[17] IGEC-2 Collaboration arXiv:0705.0688

[18] Il Nuovo Cimento 10512 (1995) 1793-1804

[19] Forward, R. L. Physical Review D 17 (2) (1978) 380-9

[20] Schutz, B. F. Class. Quantum Grav.16 (1999) A131-A156

[21] Astone, P. Physical Review Letters 91 (11) (2003) 111101

[22] Briant, T., Cerdonio, M., Conti, L., Heidmann, A., Lobo, A. and Pinard, M. Phys. Rev. D 67 (2003) 102005

[23] Keita Kawabe and the TAMA collaboration Class. Quantum Grav. 14 (1997) 1477

[24] Seichi Sato et al. Applied Optics 39 (25) (2000) 4616-4620

[25] Masaki Ando et al. Phys. Rev. Lett. 86 (18) (2001) 39504

[26] Shoemaker, D. and Schilling, R. and Schnupp, L. and Winkler, W. and Maischberger, K. and Rüdiger, A. Phys. Rev. D 32 (2) (1988) 423-432

[27] Robertson, D. I., Morrison, E., Hough, J., Killbourn, S., Meers, B. J., Newton, G. P., Robertson, N. A., Strain, K. A., Ward, H. Rev. Sci. Instrum. 66 (1995) 4447

[28] Abbott, B.P. et al. (LSC) Nucl. Instrum. Meth. Phys. Res. A 517 (2004) 154-179

[29] Willke, B. et al. Class. Quantum Grav. 19 (2002) 1377

[30] Lck, H. et al. Class. Quantum Grav. 23 (2006) S71

[31] Chelkowski, S. et al. Phys. Rev.A 71 (1) (2005) 013806

[32] D. Sigg and the LIGO Science Collaboration Class. Quantum Grav. 232006 S51

[33] Abbott, B. P. et al. Class. Quantum Grav. 24 (2007) 5343

[34] Abbott, B. P. et al. Rep. Prog. Phys. 72 (2009) 076901

[35] Kalogera, V. et al Astrophys. J. 601 (2004) L17982

[36] Abbott, B.P. for the LIGO Scientific Collaboration Astrophys. J. 681 (2008) 141928

[37] F. Acernese et al. Class. Quantum Grav. 23 (2006) S63

[38] Abbott, B.P. et al.Astrophys. J. 713 (2010) 671 
[39] Accadia, T. and Swinkels, B. L. (for the VIRGO Collaboration) Class. Quantum Grav. 27 (2010) 084002

[40] The LIGO Scientific Collaboration and the Virgo Collaboration Nature 460 (2009) 990-4

[41] Abadie, J. et al. Astrophys. J. 715 (2010) 1453

[42] Abbott, B.P. et al. Astrophys. J. 715 (2010) 1438

[43] Belczynski, K. et al Astrophys. J. 572 (2002) 407

[44] Acernese, F. et al. Journal of Physics: Conference Series 32 (2006) 223229

[45] Smith, J. R. (for the LIGO Scientific Collaboration) Class. Quantum Grav. 262009114013

[46] Sigg, D. (for the LIGO Scientific Collaboration) Class. Quantum Grav. 252008114041

[47] F Acernese et al. Class. Quantum Grav. 252008184001

[48] Willke, B. et al. Class. Quantum Grav. 23 (2006) S207

[49] Amaro-Seoane, P. et al. Einstein Telescope Design Study: Vision Document ET Codified Document ET-03009 (2009)

[50] Kazuaki Kuroda and the LCGT Collaboration Class. Quantum Grav. 232006 S215

[51] Miyoki, S. et al. Class. Quantum Grav. 21 (2004) S1173

[52] Barriga, P., Arain, M. A., Guido Mueller, G., Zhao, C.and David G. Blair, D. G. Optical design of the proposed Australian International Gravitational Observatory Opt. Express 17 (2009) 2149-65 